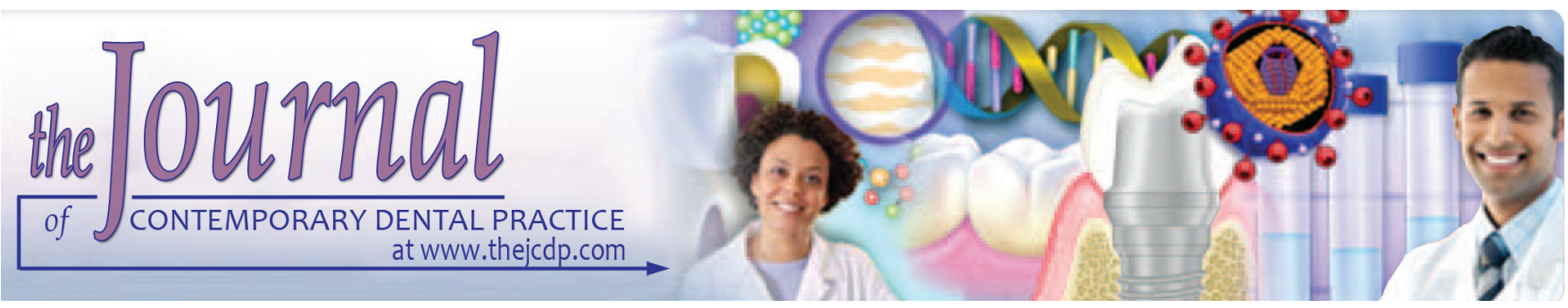

\title{
In vitro Assessment of Influence of Various Bleaching Protocols on the Strength of Ceramic Orthodontic Brackets bonded to Bleached Tooth Surface: A Comparative Study
}

\author{
${ }^{1}$ Divya Iska, ${ }^{2}$ Raghu Devanna, ${ }^{3}$ Madhvi Singh, ${ }^{4}$ Rajkiran Chitumalla, ${ }^{5}$ Sai C Bala Balasubramanian, ${ }^{6}$ Manish Goutam
}

\begin{abstract}
Introduction: Esthetics is one of the common issues because of which patients consult dental orthodontic treatment. Two ways of tooth bleaching are available these days, which includes in-office bleach and home bleach. Various bleaching protocols are available these days for treating the tooth surfaces. Hence, we planned the present study for investigating the impact of various intracoronal bleaching protocols on shear bond strength of ceramic brackets bonded to tooth surface after bleaching.

Materials and methods: The present study included assessment of 100 extracted maxillary central incisors with the integrated buccal surface. A resin block was made and individual teeth were embedded in each block. Root canal therapy procedure was performed in all the teeth, after which $2 \mathrm{~mm}$ short of tooth apex up to the level of cementoenamel junction, removal of the root canal filling was done. All the samples were broadly divided into four study groups with 25 samples in each group. Bleaching procedure was carried in all the samples intracoronally followed by testing of shear bond strength using universal force testing machine. Following the modified adhesive remnant index
\end{abstract}

\footnotetext{
${ }^{1}$ Department of Orthodontics, Sri Ramachandra Dental College and Hospital, Chennai, Tamil Nadu, India

${ }^{2}$ Orthodontic Division, Department of Preventive Dentistry, Faculty of Dentistry, Taif University, AL-Taif, Kingdom of Saudi Arabia

${ }^{3}$ Department of Periodontist, Dentica Oral Care, Ranchi Jharkhand, India

${ }^{4}$ Prosthodontics Division, Department of Oral and Maxillofacial Rehabilitation, Qaseem Private College, Buraydah, Kingdom of Saudi Arabia

${ }^{5}$ Private Practitioner, Smile Hub Dental Clinic, Chennai, Tamil Nadu, India

${ }^{6}$ Department of Prosthodontist, Dentica Oral Care, Ranchi Jharkhand, India

Corresponding Author: Divya Iska, Department of Orthodontics Sri Ramachandra Dental College and Hospital, Chennai, Tamil Nadu, India, e-mail: dr.gmadhanreddy@gmail.com
}

(Al), assessment of remaining adhesive on the brackets was done. All the results were compiled and analyzed by Statistical Package for the Social Sciences (SPSS) software version 17.0.

Results: In the control group, mean shear bond strength was found to be $17.9 \mathrm{MPa}$. While comparing the carbamide peroxide (CP) group with sodium perborate study group, we observed a statistically significant difference. Nonsignificant results were obtained while comparing the shear bond strength in between sodium perborate group and hydrogen peroxide (HP) group.

Conclusion: Intracoronal bleaching does affect the shear bond strength of ceramic brackets. Sodium perborate bleaching influences shear bond strength more strongly than other bleaching agents such as CP and HP.

Clinical significance: In patients undergoing orthodontic treatment, HP is a preferred agent where bleaching has to be followed by orthodontic bonding to the tooth surface.

Keywords: Bleaching, Ceramic, Shear bond strength.

How to cite this article: Iska D, Devanna R, Singh M, Chitumalla R, Balasubramanian SCB, Goutam M. In vitro Assessment of Influence of Various Bleaching Protocols on the Strength of Ceramic Orthodontic Brackets bonded to Bleached Tooth Surface: A Comparative Study. J Contemp Dent Pract 2017;18(12): $1181-1184$

Source of support: Nil

Conflict of interest: None

\section{INTRODUCTION}

One of the most common reasons for which patients seek dental treatment is esthetic issues and tooth staining. It has numerous complex etiologic components that are normally categorized into intrinsic and extrinsic. ${ }^{1-3}$ Two ways of tooth bleaching are available these days, which includes in-office bleach and home bleach. For the past many years, in-office bleach is being used in dentistry. Tooth structures need not be reduced while performing these bleaching procedures. ${ }^{4-6}$ 
The main state of orthodontic treatment is holding the orthodontic appliances and components to tooth surfaces. Be that as it may, this progression is impermanent, on the grounds that the brackets are removed after completion of active orthodontic treatment, and keeping up a sound, unblemished surface of enamel subsequent to debonding is the clinician's essential goal. ${ }^{7-9}$ Intracoronal bleaching is a preservationist contrasting option to more intrusive nonvital esthetic treatment, for example, placement of crowns and veneers on discolored and stained teeth. ${ }^{10,11}$ Hence, we planned the present study for investigating the impact of various intracoronal bleaching protocols on shear bond strength of ceramic brackets bonded to tooth surface after bleaching.

\section{MATERIALS AND METHODS}

The present study was conducted in the Department of Orthodontics of the dental institution and included assessment of 100 extracted maxillary central incisors with integrated buccal surface. Ethical approval was taken from the institutional ethical committee before starting of the study. Once the teeth were extracted, they were cleaned under running tap water and were kept in thymol solution until further assessment was done. A cuboidal block was made from autopolymerization resin material and individual teeth were embedded in each block. Inclusion criteria for the present study included:

- Freshly extracted maxillary central incisors

- Noncarious teeth

- Teeth that were extracted due to periodontal reasons

- Tooth with intact surface structures.

Access opening was done in all the teeth using a round carbide bur followed by biomechanical preparation. During preformation of these procedures, thorough irrigation of the root canal was done using sodium hypochlorite solution followed by drying with sterile paper points. Root canal sealer and gutta-percha points were used for obturating the root canal space. About $2 \mathrm{~mm}$ short of tooth apex up to the level of cementoenamel junction, removal of the root canal filling was done after the completion of obturation process. It was followed by application of a 2-mm-thick coating of glass ionomer cement. All the teeth specimens, after completion of obturation, were then broadly divided into four study groups with 25 samples in each group as shown in Table 1.

Table 1: Division of samples into various study groups

\begin{tabular}{lll}
\hline Group & Parameter & $n$ \\
\hline I & Composite restoration & 25 \\
II & Intracoronal bleaching: $35 \% \mathrm{CP}$ & 25 \\
III & Intracoronal bleaching: sodium perborate & 25 \\
IV & Intracoronal bleaching: $37.35 \% \mathrm{HP}$ & 25 \\
\hline
\end{tabular}

Placement of bleaching agent, according to its study group, was done into the access cavity followed by closing with the temporary filling material. Repetition of the procedure was done after 4 days. Removal of the temporary filling was done after 2 weeks' time followed by rinsing of the access cavity with distilled water. After rinsing, restoration of the tooth sample was done with the composite restorative material. This was followed by immersion of the teeth in artificial saliva for 1 month before bracket removal. Thorough cleaning of the teeth samples was done after debonding, with nonfluorinated pumice and distilled water. Afterward, the teeth specimens were dried with compressed air; 37\% phosphoric acid gel application was done on the buccal surface of tooth specimen for 15 seconds. This was followed by rinsing and drying. After completion of the etching process, application of a uniform coating of primer (Grasim Bleaching Ltd., India) was done on the etched enamel surface followed by curing for 5 seconds. Orthodontic ceramic brackets (Class One Orthodontics, India) were used for bonding to the cured enamel surface. Removal of the excessive adhesive solution was done. Universal forces testing machine was used for calculating the shear bond strength of each specimen. Stereomicroscopic examination of individual tooth was done after debonding the brackets. Following the modified AI, the assessment of remaining adhesive on the brackets was done. ${ }^{12}$ All the results were compiled and analyzed by SPSS software version 17.0. Chi-square test, one-way analysis of variance (ANOVA), and Tukey honest significant difference multiple comparison tests were used for assessment of the level of significance; $\mathrm{p}<0.05$ was taken as statistically significant.

\section{RESULTS}

We divided freshly extracted 100 maxillary central incisors into four study groups (Table 1). Mean shear bond strength observed in the control group was $17.9 \mathrm{MPa}$ (Table 2). In the study groups I, II, and III, the mean shear bond strength was found to be 15.10, 13.02, and $15.56 \mathrm{MPa}$, respectively (Graph 1 ). While comparing the CP group (group II) with sodium perborate study group (group III), ANOVA suggested that statistically significant difference $(\mathrm{p}<0.05)$ exists between them (Table 3$)$. Nonsignificant results were obtained while comparing the shear bond strength in between sodium perborate

Table 2: Mean shear bond strength of specimens of different study groups

\begin{tabular}{lll}
\hline Group & Mean shear bond strength (MPa) & Standard deviation \\
\hline I & 17.99 & 1.6251 \\
II & 15.10 & 1.7958 \\
III & 13.02 & 1.3622 \\
IV & 15.56 & 1.1826 \\
\hline
\end{tabular}




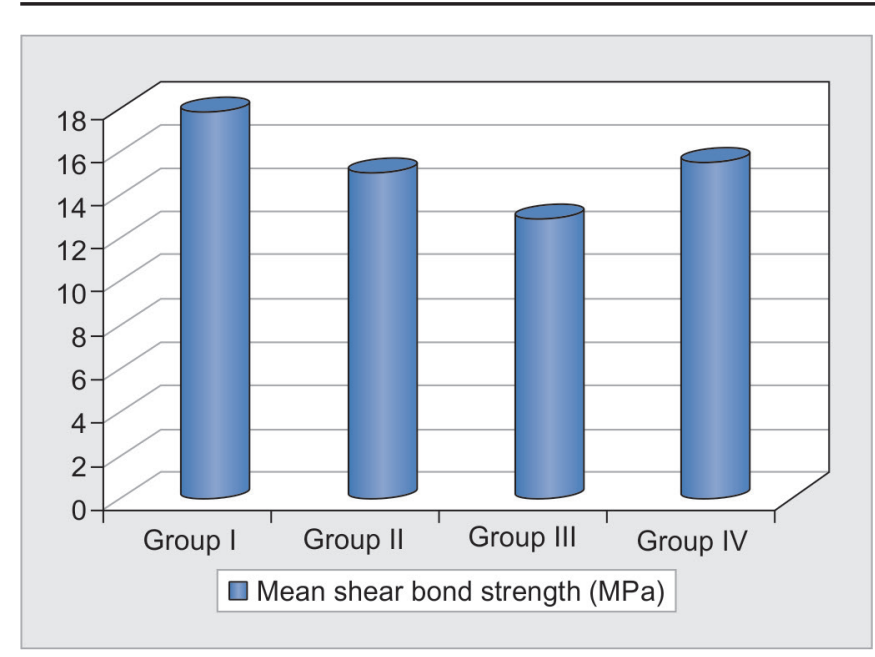

Graph 1: Comparison of mean shear bond strength of specimens of different groups

Table 3: Comparative evaluation of mean shear bond strength in between different study groups (ANOVA statistical test)

\begin{tabular}{lll}
\hline Study groups & Standard error & $p$-value \\
\hline Group I vs II & 0.55896 & $0.03^{*}$ \\
Group I vs III & 0.55896 & $0.02^{*}$ \\
Group I vs IV & 0.55896 & $0.01^{*}$ \\
Group II vs III & 0.55896 & $0.03^{*}$ \\
Group II vs IV & 0.55896 & 0.10 \\
Group III vs IV & 0.55896 & $0.02^{*}$ \\
Intergroup comparison & - & $0.04^{*}$ \\
\hline
\end{tabular}

*Significant

group and HP group $(\mathrm{p}>0.05)$. Description of AI scores in different study groups is depicted in Graph 2. Majority of specimens in groups II, III, and IV exhibited AI score 3.

\section{DISCUSSION}

One of the important steps involved in commencing orthodontic treatment is the bonding of orthodontic brackets to the tooth surface. This is accompanied by removal of brackets after completion of orthodontic treatment. Lots of controversies exist in relation to the effect of different bleaching protocol on the shear bond strengths of brackets. ${ }^{13,14}$ Hence, we planned the present study for investigating the impact of various intracoronal bleaching protocols on shear bond strength of ceramic brackets bonded to tooth surface after bleaching.

In the present study, we analyzed a total of 100 extracted tooth specimens by dividing them randomly into four study groups divided based on the bleaching protocol followed (Table 1). Control group followed by HP group exhibited maximum shear bond strength (Table 2 and Graph 1). We observed statistically significant difference on comparing the mean shear bond strength in between various study groups (Table 3). Our results were in correlation with the results obtained by Chauhan et $\mathrm{al}^{15}$ who also reported similar findings in their study. Oztaş

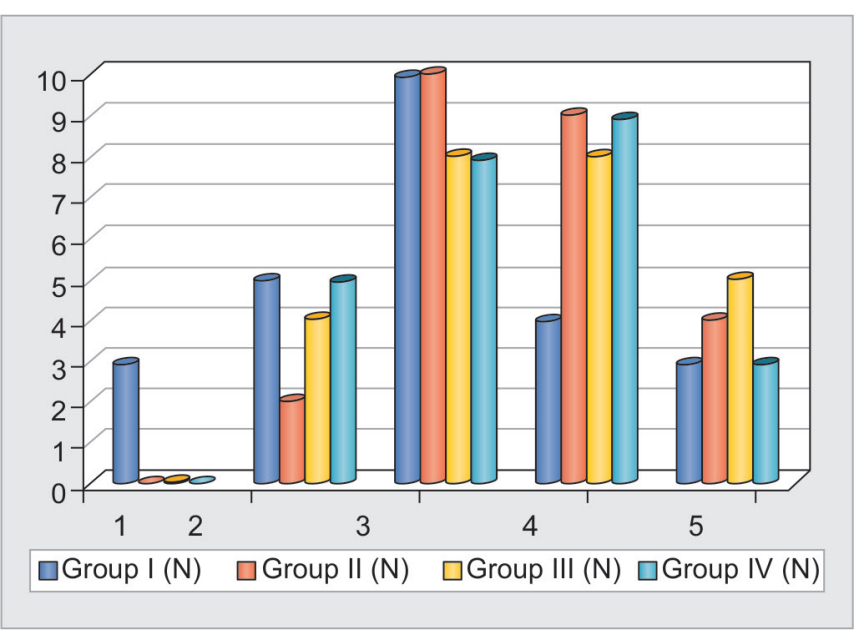

Graph 2: Description of RI scores for different study groups

et $\mathrm{a}^{16}$ assessed the impact of bleaching and late bonding on the shear bond strengths of metal and ceramic brackets bonded with light and chemically cured composite resin to human teeth. They analyzed a total of 120 freshly extracted human premolars and divided them randomly into three study groups with 40 specimens in each group. Bleaching in first group was done by $20 \%$ $\mathrm{CP}$ while in the third group, no bleaching procedure was carried, which was taken as control. Division of the main groups was done into subgroups as per various adhesivebracket combinations. In groups I and II, bonding to the specimens was done after 1 and 14 days, respectively. Universal testing machine was used for debonding all the specimens, and modified AI was used for assessing the fracture properties. They did not observe any significant difference while comparing the shear bond strength of metal and ceramic brackets. They also observed failure at bracket/adhesive interface. From the results, they concluded that shear bond strength is unaffected by $20 \% \mathrm{CP}$.

Impact of various methods of bleaching on the shear bond strength of ceramic brackets was assessed by Chauhan et al. ${ }^{15}$ They analyzed 60 freshly extracted maxillary central incisors and randomly divided into four study groups with 15 specimens in each group. They prepared the access cavity in all the extracted teeth, and after completing the root canal process, they removed the filling from cementoenamel junction till $2 \mathrm{~mm}$ short of apex. They performed intracoronal bleaching with $35 \% \mathrm{CP}$, sodium perborate, and $37.5 \% \mathrm{HP}$ in groups II, III, and IV respectively. Afterward, they immersed the teeth in artificial salivary solution for 1 month before debonding it. This was followed by bonding of the tooth surface with ceramic brackets. Shear bond strength was tested after bonding, using universal force testing machine. They observed statistically significant results in between all the study groups except in between groups II and IV. 
Furthermore, they found no significant difference in terms of AI score in between various study groups.

While assessing the mean AI scores in different study groups, we observed that in HP group (group IV), bond failure occurred in the area in vicinity of enamel/adhesive interface (Graph 2). Similar results have been reported previously in the literature. ${ }^{15}$ Influence of intracoronal bleaching agents and protocols on the shear bond strength of orthodontic brackets was assessed by Gungor et al. ${ }^{3}$ They evaluated 60 freshly extracted mandibular incisors and divided them randomly into four study groups with 15 specimens in each group. After performing root canal therapy in each group, they removed the fillings from $2 \mathrm{~mm}$ short of apex till cementoenamel junction and applied zinc phosphate base. While on the one hand, Group 1 served as control, in groups II, III, and IV, HP, sodium perborate, and $37 \% \mathrm{CP}$ were used as bleaching agent respectively. This was followed by curing of orthodontic brackets on the etched enamel surface of teeth of all the study groups. They tested the shear bond strength of all the specimens using universal testing machine. In comparison to the control group, they observed significantly lower shear bond strength in all the study groups. They concluded that shear bond strength of orthodontic brackets is significantly affected by intracoronal bleaching agents.

\section{CONCLUSION}

Shear bond strength of ceramic brackets is significantly influenced by intracoronal bleaching. This effect persists even 1 month after the bleaching process. In comparison to others, sodium perborate bleaching had a maximum negative effect.

\section{REFERENCES}

1. Gungor AY, Ozcan E, Alkis H, Turkkaharaman H. Effects of different bleaching methods on shear bond strengths of orthodontic brackets. Angle Orthod 2013 Jul;83(4):686-690.

2. Uysal T, Er O, Sagsen B, Ustdal A, Akdogan G. Can intracoronally bleached teeth be bonded safely? Am J Orthod Dentofacial Orthop 2009 Nov;136(5):689-694.

3. Gungor AY, Ozcan E, Alkis H, Turkkahraman H. Effects of different intracoronal bleaching methods on shear bond strengths of orthodontic brackets. Angle Orthod 2012 Sep;82(5): 942-946.

4. Ferreira NS, Rosa PC, Ferreira RD, Valera MC. Evaluation of shear bond strength of orthodontic brackets bonded on the tooth surface after internal bleaching. Rev Odontol UNESP 2014 May-Jun;43(3):209-213.

5. Natt AS, Sekhon AK, Munjal S, Dggal R, Holla A, Gupta P, Gandhi P, Sarin S. A comparative evaluation of static frictional resistance using various methods of ligation at different time intervals: an in vitro study. Int J Dent 2015 Nov;2015:407361.

6. Miles PG, Pontier JP, Bahiraei D, Close J. The effect of carbamide peroxide bleach on the tensile bond strength of ceramic brackets: an in vitro study. Am J Orthod Dentofacial Orthop 1994 Oct;106(4):371-375.

7. Uysal T, Sisman A. Can previously bleached teeth be bonded safely using self-etching primer systems? Angle Orthod 2008 Jul;78(4):711-715.

8. Wang L, Francisconi LF, Atta MT, dos Santos JR, Del Padre NC, Gonini A Jr, Fernandes KBP. Effect of bleaching gels on surface roughness of Nano filled composite resins. Eur J Dent 2011 Apr;5(2):173-179.

9. Oskoee PA, Kahnamoui MA, Oskoee SS, Zadfattah F, Pournaghi-Azar F. Effects of in-office and home bleaching gels on the surface mercury levels of dental amalgam. Eur J Dent 2010 Jan;4(1):23-27.

10. Haywood VB, Heymann HO. Night guard vital bleaching. Quintessence Int 1989 Mar;20(3):173-176.

11. Cacciafesta V, Sfondrini MF, Stifanelli P, Scribante A, Klersy C. The effect of bleaching on shear bond strength of brackets bonded with a resin-modified glass ionomer. Am J Orthod Dentofacial Orthop 2006 Jul;130(1):83-87.

12. Olsen ME, Bishara SE, Damon P, Jakobsen JR. Evaluation of scotch bond multipurpose and maleic acid as alternative methods of bonding orthodontic brackets. Am J Orthod Dentofacial Orthop 1997 May;111(5):498-501.

13. Torneck CD, Titley KC, Smith DC, Adibfar A. The influence of time of hydrogen peroxide exposure on the adhesion of composite resin to bleached bovine enamel. J Endod 1990 Mar;16(3):123-128.

14. Zachrisson $Y O$, Zachrisson BU, Buyukyilmaz T. Surface preparation for orthodontic bonding to porcelain. Am J Orthod Dentofacial Orthop 1996 Apr;109(4):420-430.

15. Chauhan V, Kumar P, Sharma P, Shetty D. Effect of different intracoronal bleaching methods on shear bond strength of ceramic brackets bonded to bleached enamel: an in-vitro study. J Orthod Sci 2017 Jul-Sep;6(3):86-90.

16. Oztaş E, Bağdelen G, Kiliçoğlu H, Ulukapi H, Aydin I. The effect of enamel bleaching on the shear bond strengths of metal and ceramic brackets. Eur J Orthod 2012 Apr;34(2):232-237. 